\title{
Politique
}

Politique

\section{Diane Bellemare et Céline Saint-Pierre (sous la direction de), Les stratégies de reprise, Montréal, Éditions Albert Saint-Martin, 1984, 252 p.}

\section{Raymond Hudon}

Numéro 9, hiver 1986

Démocratie et libéralisme

URI : https://id.erudit.org/iderudit/040529ar

DOI : https://doi.org/10.7202/040529ar

Aller au sommaire du numéro

Éditeur(s)

Société québécoise de science politique

ISSN

0711-608X (imprimé)

1918-6584 (numérique)

Découvrir la revue

Citer ce compte rendu

Hudon, R. (1986). Compte rendu de [Diane Bellemare et Céline Saint-Pierre (sous la direction de), Les stratégies de reprise, Montréal, Éditions Albert Saint-Martin, 1984, 252 p.] Politique, (9), 209-215.

https://doi.org/10.7202/040529ar

Ce document est protégé par la loi sur le droit d'auteur. L'utilisation des services d'Érudit (y compris la reproduction) est assujettie à sa politique d'utilisation que vous pouvez consulter en ligne.

https://apropos.erudit.org/fr/usagers/politique-dutilisation/ 
Diane Bellemare et Céline Saint-Pierre (sous la direction de), Les stratégies de reprise, Montréal, Éditions Albert Saint-Martin, 1984, $252 \mathrm{p}$.

Dans leur introduction à ce recueil de la plupart des communications présentées au troisième colloque de l'Association d'économie politique, Diane Bellemare et Céline Saint-Pierre signalent que depuis ce moment, «les journaux font état d'une prétendue 
reprise économique» (p. 13). Un certain pessimisme ferait ressortir le caractère incertain de cette reprise. Il faudrait par ailleurs grandement embellir la situation pour ne pas souligner que «la reprise économique dont on parle n'est pas une reprise pour l'ensemble de ceux et celles qui travaillent ou qui désirent travailler » (p. 16). Ceci dit, ce n'est peut-être pas tant le caractère d'incertitude de la reprise que les effets inégalement ressentis de la crise qu'il convient de soumettre a un examen plus attentif.

Malgré qu'il se révèle couramment plus facile de mesurer l'ampleur de la crise (et de la reprise) à l'aide d'indicateurs quantitatifs relativement simples, parfois simplement bêtes (taux de chômage, taux d'inflation, rythme de faillites, etc.), il faut tout de même rappeler que toute crise économique traduit ou se prolonge dans la transformation profonde des rapports sociaux et politiques. Michel Jurdant, pour sa part, s'insurge contre l'acceptation forcée ( "on n'a pas le choix») et même l'attitude de résignation qui semblent marquer la révolution sociale que la crise permet d'imposer. Selon lui, la seule alternative d'un "virage écologique» se présente pour contrer "la dictature technologique» qui est en train de s'implanter, mettant en présence «ceux qui la font [les experts]: scientifiques et technocrates, et ceux qui la subissent [les citoyens]: les non-experts ou les nouveaux prolétaires» (p. 24).

Les analyses présentées dans Les stratégies de reprise renforcent en grande partie les charges dénonciatrices de Jurdant contre l'autoritarisme de la science, "piège dans lequel sont tombées toutes les religions» (p. 223). Le thème de l'inévitabilité se trouve en effet repris par quelques intervenants au colloque, même si à la manière de Gösta Rehn, il est alors précisé que l'on ne veut que "présenter une perspective à plus court terme», disant avec insistance que «vouloir créer la solidarité totale constitue un projet à plus long terme» (p. 239). Il faut cependant bien voir que le projet de solidarité dont parle Rehn a un caractère national prononcé, 
du moins si l'on se reporte à la présentation qu'il fait de l'expérience suédoise: «La politique active du marché du travail mise en œuvre par la Suède a pour but de dépasser les conflits d'objectifs qui peuvent surgir entre [les syndicats et les employeurs]» (p. 133). Les politiques de l'Administration nationale du travail pourraient en fait constituer un beau cas d'illustration d'une politique de coopération forcée (Rehn parle de coopération mutuelle.) à travers laquelle «les intérêts des deux parties peuvent [...] être servis».

La question demeure quand même de savoir si ces intérêts sont satisfaits également. À tout le moins quelques analyses regroupées dans la publication dirigée par Bellemare et Saint-Pierre contribuent-elles à confirmer les inégalités engendrées, régulièrement amplifiées, par les transformations accompagnant la révolution technologique. En examinant les phénomènes de la restructuration du marché du travail et de la recomposition de la force de travail dans le secteur tertiaire, Céline Saint-Piere est amenée à poser que «les changements technologiques rapides et massifs qui s'opèrent actuellement nous permettent de comprendre qu'il n'est plus possible d'assimiler ou de confondre la notion de développement technologique avec celle de progrès social» (p. 111). Dès lors, il se révèle pertinent de noter, d'après les données analysées par Colette Bernier, qu'entre 1975 et 1982, "l'emploi à temps partiel au Québec est passé de $7 \%$ à plus de $12 \%$ de la population active» (p. 87) et que «la plus large part du travail à temps partiel continue à relever des entreprises du tertiaire» (p. 94).

Il est imaginable que soit décelée à travers cette tendance "une politique à plus long terme visant une plus grande flexibilité dans la gestion des capacités de travail» (p. 87). Le succès de cette politique, en autant qu'on puisse l'estimer vraiment délibérée, puise peut-être paradoxalement au fait que «la source d'identité individuelle et sociale est de moins en moins basée sur l'éthique 
du travail, et cela pour un nombre croissant d'individus» (p. 142). Cette «désaffectation vis-à-vis du travail salarié» que Paul Grell retrace à travers des sondages faits dans différents pays n'est sans doute pas tout à fait imaginaire. Mais n'est-il pas approprié de remarquer une désaffectation probablement aussi importante visà-vis le politique, parce que justement il ne se montre pas suffisamment en mesure d'assurer des conditions propices à la croissance ou même simplement au maintien de l'emploi? Entre différentes visions du monde, entre l'aspiration plus ou moins vérifiable et la nécessité plus ou moins acceptée, que de place pour le discours parfois paré d'analyse. À tout le moins, un vaste espace apparaîtil disponible pour l'ambivalence...

Cette ambivalence est repérable à travers les présentations faites autour de la restructuration industrielle qui résulte de la crise et qui traverse les stratégies de reprise. Ainsi, David Wolfe ne peut s'exprimer plus justement en proposant: "The growing trend towards deindustrialization is becoming one of the most hotly debated issues in Canada» (p. 202). Car Philippe Faucher et Robert Young jugent avec clarté que «le terme de désindustrialisation ne convient pas pour désigner [l'évolution structurelle de l'industrie canadienne]». En effet, pour rendre compte du fait qu' «au Canada, comme dans l'ensemble des pays de l'O.C.D.E., à l'exception de la Grande-Bretagne, la valeur de la production industrielle n'a cessé d'augmenter, $[. .$.$] le terme de déclin industriel$ convient mieux [pour traduire] la diminution relative de la contribution du secteur manufacturier au produit intérieur» (p. 171). Faucher et Young mesurent avec précision et évaluent la portée de ce déclin qui se poursuit depuis dix ans déjà (p. 174). Leur contribution peut être considérée grandement pertinente, à l'heure précisément où le libre échange est inscrit à l'order du jour politique canadien, surtout qu'ils rappellent le très grand libéralisme marquant déjà la politique commerciale du Canada (p. 193). 
Admettons, l'ambivalence de l'analyse peut n'être que répercussion de l'ambivalence-ambiguïté de l'action analysée. Ce problème semble spécialement présent dans toute discussion sur le devenir de l'État-providence au Canada. Aussi Allan Moscovitch est-il conduit à conclure que, «même si les principaux représentants de l'État et du Capital sont mal à l'aise devant le maintien de la prédominance de l'idéologie et de la pratique de l'État-providence, les personnes qui proposent les doctrines de la nouvelle droite radicale n'ont pas réussi à s'assurer une position dominante. La restructuration du régime de sécurité sociale a donc été marquée par l'ambiguïté, les principes de l'universalité et de la sélectivité étant invoqués simultanément» (p. 43). Malgré la détermination un temps affichée par le gouvernement conservateur installé à Ottawa dans sa volonté de trancher dans le sens de la sélectivité, Diane Bellemare n'aurait sans doute pas à investir jusqu'à ses dernières énergies pour maintenant convaincre les dirigeants de ce gouvernement que «la crise de régulation ne peut [pas facilement] anéantir les forces qui ont poussé à la création des régimes publics de sécurité du revenu et cela, tant et aussi longtemps que le régime salarial constituera le mécanisme privilégié de répartition du revenu» (p. 56).

Le problème serait-il, comme le suggère Carolle Simard, que l'on a confondu «crise de l'État-providence et bonne santé de la bureaucratie» (p. 78)? Après tout, les programmes de privatisation de l'État peuvent pratiquement équivaloir à une «diffusion de l'appareil bureaucratique», les organismes autonomes privés n'échappent pas au modèle bureaucratique tout comme il est devenu de plus en plus périlleux de distinguer sur ce point les organismes du secteur public «de ceux du secteur privé». Les objectifs de décentralisation et de privatisation véhiculés par l'idéologie du libéralisme ne parviennent pas ainsi à dissimuler le paradoxe de «l'illusion de la déperdition des contrôles [qui se 
trouvent] néanmoins consolidés à travers des relais de tous ordres » (p. 77).

Force est de constater que les idéologies conservatrices ne sont pas (encore) venues à bout complètement de l'État-providence. Selon Suzanne de Brunhoff, leur fonction pratique n'est cependant pas totalement insignifiante: «Elles évacuent le problème du chômage tel qu'il était posé dans la tradition keynésienne, qui considérait l'action étatique par rapport au niveau de l'emploi et du revenu dans un pays. De cette façon elles reflètent certains aspects de la restructuration des emplois au cours de la crise, et justifient les pratiques de délocalisation, transferts, précarisation, etc» (p. 67). L'épuisement du modèle keynésien auquel se réfere aussi Pierre Rosanvallon impose d'une certaine manière "la recherche d'effets équivalents au niveau micro-économique [qui] passe par une redéfinition du rôle et de la forme des acteurs sociaux, et notamment de l'acteur syndical» (p. 164).

$\mathrm{Si}$, selon Rosanvallon, les syndicats craignent d'abord la remise en cause de leur fonction collective, ils doivent se montrer tout autant préoccupés par un approfondissement et une extension de la fonction de relais qu'ils ont toujours exercée à des degrés variables et avec une conscience plus ou moins grande. Il est toutefois remarquable que quelques expériences de gouvernement de gauche (ou moins de droite) aient contribué à l'accélération de ce processus. Le courant conservateur qui, selon de Brunhoff, a marqué «de son empreinte beaucoup d'analyses, y compris à gauche» (p. 68), n’a certainement pas épargné beaucoup de gouvernements, guidés et obsédés, de droite à gauche, par les impératifs d'une inéluctable («on n'a pas le choix») rationalisation.

Dans de telles conditions, le cynisme ne laisserait place qu'aux aveux (lucides?) d'impuissance. À lire Les stratégies de reprise, on acquiert la nette impression que les forces de changement ne se concentrent pas du côté de l'opposition. L'alternative qui semble 
se dégager ressemble à la charge, à coup de manifeste. Les alliances possibles pour traduire cela en action apparaissent par ailleurs se raréfier. Les textes contenus dans Les stratégies de reprise permettent, au moins pour une partie non négligeable, de mieux saisir ce qui est en train de s'opérer comme processus de réorganisation sociale et économique, tout en tentant de ne pas adopter servilement le point de vue de ceux qui se trouvent actuellement aux commandes.

Raymond

Hudon

Université Laval 